\title{
UTILIZAÇÃO DA FIBRA DO OURICURI (Syagrus coronata) NA REMOÇÃO DO CORANTE AZUL DE METILENO: VARIÁVEIS DE PROCESSO E ISOTERMA DE ADSORÇÃO
}

\author{
T. S. SILVA ${ }^{1}$, D. C. HENRIQUE ${ }^{1}$, L. MEILI ${ }^{1 *}$, J. I. SOLETTI ${ }^{1}$, S. H. V. CARVALHO ${ }^{1}$ \\ ${ }^{1}$ Laboratório de Sistemas de Separação e Otimização de Processos, Centro de Tecnologia \\ Universidade Federal de Alagoas \\ *e-mail: lucas.meili@ctec.ufal
}

\begin{abstract}
RESUMO
Neste trabalho foi avaliada a capacidade de adsorção da fibra do ouricuri (Syagrus coronata) "in natura" na remoção do corante azul de metileno, sendo empregado o planejamento experimental $2^{3}$ que demonstrou a influência significativa das variáveis de processo: massa de adsorvente $(\mathrm{M})$, granulometria $(\mathrm{G})$ e agitação $(\mathrm{A})$. A partir da faixa estudada no planejamento experimental, é possível confirmar que a maior resposta, $\mathrm{q}_{\mathrm{t}}=7,7 \mathrm{mg} / \mathrm{g}$, foi alcançada utilizando $0,5 \mathrm{~g}$ de massa, granulometria de $0,595 \mathrm{~mm} \mathrm{e}$ agitação de $200 \mathrm{rpm}$. O estudo cinético demonstrou que a adsorção entre o corante azul de metileno e a fibra do ouricuri ocorre rapidamente, em torno 5 minutos, atingindo $90 \%$ da remoção da cor da solução. A isoterma realizada na temperatura de $25^{\circ} \mathrm{C}$ apresentou um $\mathrm{q}_{\mathrm{e}}=29,8 \mathrm{mg} / \mathrm{g}$, sendo os dados experimentais ajustados adequadamente ao modelo de isoterma de Sips com o $\mathrm{R}^{2}=0,985$ e $\mathrm{q}_{\text {máx }}=31,7 \mathrm{mg} / \mathrm{g}$. Os resultados demonstraram que o resíduo agroindustrial utilizado é um atrativo na remediação do corante em estudo, tanto do ponto de vista de eficiência do processo quanto em relação ao seu baixo custo.
\end{abstract}

\section{INTRODUÇÃO}

O Azul de Metileno é um corante básico, catiônico, solúvel em água e álcool e comumente utilizado pelas indústrias têxteis. Devido à presença de substâncias tóxicas, o descarte de corantes no meio ambiente é preocupante, pois os efeitos adversos gerados, tanto para o meio ambiente quanto para os seres humanos, podendo causar queimaduras nos olhos, prejudicar o sistema respiratório e, se ingerido pode causar náuseas, vômitos, sudorese e confusão mental (TAN et al., 2008). Segundo a resolução 357 do Conselho Nacional do Meio Ambiente (CONAMA), não é permitida a presença de corantes provenientes de fontes antrópicas que não sejam removíveis por processo de coagulação, sedimentação e filtração convencionais

Dessa maneira há uma grande necessidade de tratar o efluente contaminado com este composto,a partir da utilização de técnicas de baixo custo e que tenham uma ótima eficiência. Algumas tecnologias de tratamento como os processos de coagulação química, oxidação química, fotodegradação, filtração por meio de membranas, troca iônica e a adsorção são utilizadas para remoção de corantes dos efluentes (BORBA et al., 2012; GULER e SARIOGLU, 2013).

$\mathrm{O}$ processo de adsorção vem se destacando como um método eficiente na remoção de corantes. A adsorção é um processo de separação preferencial de moléculas presentes em um fluido por meio 
da sua fixação em um sólido (YAGUB et al., 2014). As vantagens dessa técnica envolvem baixo custo, facilidade de operação, não resulta na formação de substâncias nocivas e seu método é eficiente do ponto de vista ambiental em comparação aos tratamentos convencionais (AHMAD et al., 2009; TANYILDIZI, 2011).

A utilização de adsorventes de baixo custo faz com que o processo de adsorção se torne economicamente mais atraente (GAO et al., 2011). Segundo Brito et al. (2010), para um material ser considerado de baixo custo é necessário que o mesmo exija pouco processamento, seja abundante na natureza ou subproduto ou resíduo material de uma indústria.

Dentre os adsorventes de baixo custo que podem ser utilizados em processos de adsorção se destacam os resíduos agroindustriais, como a fibra do Ouricuri. A Figura 1 apresenta o fruto da palmeira do ouricuri (Syagrus coronata), uma das principais espécies de palmeiras da região semi-árida do nordeste brasileiro. A partir do fruto do ouricuri é extraído o óleo, podendo a casca e a fibra serem utilizadas como fonte de energia, artesanato e na remoção de poluentes das águas residuais (DONATO, 2005).

Figura 1 - Fruto oriundo da palmeira do ouricuri.
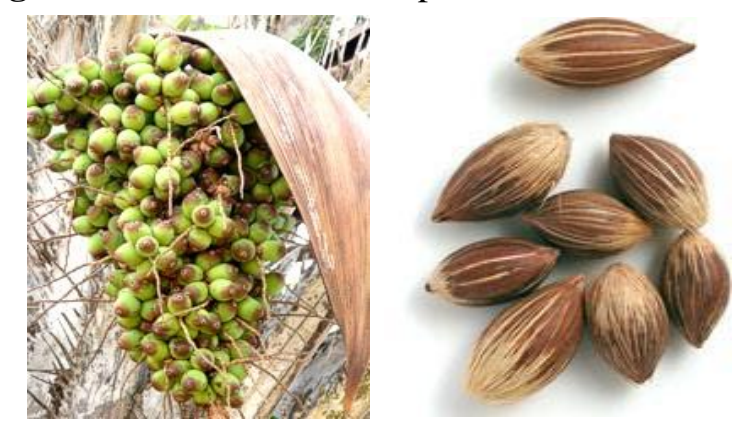

Fonte: http://www.agrocim.com.br/ (2010).

O objetivo deste trabalho foi investigar a partir do sistema em banho finito as variáveis de processos que são fundamentais na técnica de adsorção, como massa de adsorvente, velocidade de agitação e granulometria, utilizando o planejamento experimental fatorial $2^{3}$ completo. $\mathrm{O}$ estudo da isoterma de adsorção também foi realizado, sendo ajustados os modelos de isotermas disponíveis na literatura, com o intuito de verificar as características da adsorção do corante azul de metileno na fibra do ouricuri.

\section{MATERIAIS E MÉTODOS}

\subsection{Adsorvato}

Neste trabalho foi utilizado como adsorvato o corante azul de metileno, com forma molecular $\mathrm{C} 16 \mathrm{H} 18 \mathrm{~N} 3 \mathrm{SCl}$, e estrutura molecular apresentada na Figura 2.

Figura 2 - Estrutura molecular do azul de metileno.<smiles>CN(C)c1ccc2nc3ccc(N(C)C)cc3[s+]c2c1</smiles>

Fonte: Hameed et al. (2008).

As leituras de absorvância determinadas pelo espectrofotômetro UV-Vísivel foram realizadas no comprimento de onda de 665 $\mathrm{nm}$ para o corante azul de metileno, sendo utilizado para realizar a curva de calibração e determinar as concentrações do corante durante o estudo de adsorção (PAZ et al., 2013).

\subsection{Adsorvente}

A fibra do ouricuri foi utilizado como material adsorvente em sua forma "in natura". Inicialmente, o material foi lavado com água destilada e, em seguida, foi colocado para secar em estufa a uma temperatura de $60^{\circ} \mathrm{C}$ por 24 horas. Após seca, a fibra do ouricuri foi moída utilizando moinho de facas tipo Willye com peneiras de 30 e 20 mesh, para a 
obtenção das granulometrias 0,595 e 0,841 $\mathrm{mm}$, respectivamente.

Vale salientar que não há presença de óleo na fibra, e sim na amêndoa da fruto. No presente trabalho não foi realizada caracterização física do material, apenas foi estudada a sua funcionalidade como material adsorvente.

\subsection{Planejamento Experimental}

O processo de adsorção depende de vários fatores para alcançar a eficiência do processo, tais como: $\mathrm{pH}$, massa de adsorvente, velocidade da agitação, concentração do adsorvato em solução, tempo de contato, granulometria e temperatura. Todos esses fatores exercem um efeito sobre a variável resposta que pode ser quantificado com base em ensaios estatisticamente planejados, através da técnica de planejamento experimental fatorial.

Neste estudo foi realizado um planejamento experimental fatorial completo $2^{3}$, onde foram definidas as variáveis de entrada e os níveis dessas variáveis de entrada, para a elaboração da matriz de planejamento com as possíveis combinações desses níveis. A variável resposta foi a capacidade de adsorção $\left(\mathrm{q}_{\mathrm{e}}\right)$. A Tabela 1 apresenta as variáveis de entrada e os níveis utilizados no planejamento experimental fatorial completo $2^{3}$.

Tabela 1 - Variáveis de entrada e níveis utilizadas no planejamento experimental $2^{3}$.

\begin{tabular}{|l|c|c|}
\cline { 2 - 3 } \multicolumn{1}{c|}{} & \multicolumn{2}{c|}{ Níveis } \\
\hline Variáveis & $(-1)$ & $(+1)$ \\
\hline Massa (g) & 0,5 & 1,5 \\
\hline Granulometria (mm) & 0,595 & 0,841 \\
\hline Agitação (rpm) & 50 & 200 \\
\hline
\end{tabular}

Fonte: Autor (2015).

O planejamento experimental fatorial foi realizado para definir condições ótimas para a faixa estudada, sendo o experimento conduzido em banho finito, onde foi adicionado aos frascos $50 \mathrm{ml}$ da solução do corante azul de metileno com concentração inicial de $100 \mathrm{mg} . \mathrm{L}^{-1}$ e, na sequência, foi colocada a massa de adsorvente. A mistura corante e adsorvente foi deixada sob agitação constante na incubadora shaker a uma temperatura média de $25^{\circ} \mathrm{C}$ e tempo de contato de 180 minutos, sendo este o tempo de equilíbrio já verificado a partir de experimentos anteriores. Após o término deste tempo, foi retirada uma alíquota da mistura (sem a presença do adsorvente), e realizada a leitura no espectrofotômetro, no comprimento de onda de $665 \mathrm{~nm}$, para determinação concentração final do corante azul de metileno em solução.

Os experimentos foram realizados em duplicata, de forma aleatória e a resposta estudada foi a capacidade de adsorção $\left(\mathrm{q}_{\mathrm{e}}\right)$, determinada pela Equação 1.

$q_{e}=\frac{C_{0}-C_{e}}{M} \cdot V$

$\mathrm{Na}$ qual $\mathrm{C}_{0}$ é a concentração inicial do adsorvato $\left(\mathrm{mg} . \mathrm{L}^{-1}\right), \mathrm{C}_{\mathrm{e}}$ é a concentração do adsorvato no equilíbrio $\left(\mathrm{mg}^{\mathrm{L}} \mathrm{L}^{-1}\right), \mathrm{V}$ é o volume da solução (L), M é a massa de adsorvente $(\mathrm{g})$ e $\mathrm{q}_{\mathrm{e}}$ é a quantidade adsorvida no equilíbrio (mg.g $\left.{ }^{-1}\right)$.

\subsection{Cinética de Adsorção}

A avaliação da cinética de adsorção do corante azul de metileno a partir a fibra do ouricuri "in natura" foi obtida variando o tempo de contato entre o adsorvente e o adsorvato $(0,5$ - $60 \mathrm{~min})$, concentração inicial da solução de $100 \mathrm{mg} . \mathrm{L}^{-1}$ e temperatura de $25^{\circ} \mathrm{C}$. Os valores das variáveis utilizadas no estudo cinético (massa de adsorvente, granulometria e velocidade de agitação) foram as que obtiveram os melhores resultados no planejamento experimental. Os experimentos foram realizados em duplicata, sendo o procedimento de adsorção similar ao detalhado no item 2.3. 
O \% Remoção do corante foi calculado conforme Equação 2:

$\% \operatorname{Re} m o c ̧ \tilde{a} o=\frac{C_{0}-C_{f}}{C_{0}} \cdot 100$

$\mathrm{Na}$ qual $\mathrm{C}_{0}$ é a concentração inicial do adsorvato $\left(\mathrm{mg} . \mathrm{L}^{-1}\right)$ e $\mathrm{C}_{\mathrm{f}}$ é a concentração final da solução $\left(\mathrm{mg} \cdot \mathrm{L}^{-1}\right)$.

\subsection{Isoterma de Adsorção}

A isoterma de adsorção do corante azul de metileno para a fibra do ouricuri "in natura" foi obtida variando a concentração inicial das soluções $\left(5\right.$ - $\left.450 \mathrm{mg} . \mathrm{L}^{-1}\right)$ e a temperatura foi mantida constante em torno de $25^{\circ} \mathrm{C}$. Os valores das variáveis utilizadas no estudo da isoterma (massa de adsorvente, granulometria e velocidade de agitação) foram as que obtiveram os melhores resultados no planejamento experimental. Os experimentos foram realizados em duplicata, sendo o procedimento de adsorção similar ao detalhado no item 2.3.

Os dados experimentais obtidos foram aplicados aos modelos de isoterma de Langmuir, Freundlich, Sips e RedlichPeterson.

\subsubsection{Isoterma de Langmuir}

Langmuir propôs uma teoria para explicar a adsorção sobre uma superfície uniforme, simples, finita e não porosa (LANGMUIR, 1918). O modelo de Langmuir assume que a adsorção ocorre em monocamada, ou seja, cada sítio pode acomodar somente uma entidade adsortiva. Conforme Gimbert et al. (2008), a energia de adsorção é constante e não depende do grau de ocupação dos sítios ativos do adsorvente. Sendo que depois de ocupado um sítio, não pode ter mais adsorção naquele local. Esses sítios são idênticos e energeticamente equivalentes, e não há interação entre as moléculas adsorvidas sobre os sítios vizinhos. A expressão da isoterma de Langmuir é representada pela Equação 3 (LANGMUIR, 1918):

$q_{e}=\frac{q_{\max } \cdot K_{L} \cdot C_{e}}{1+K_{L} \cdot C_{e}}$

Onde qmáx é a quantidade máxima de cobertura em monocamada $\left(\mathrm{mg}^{-\mathrm{g}^{-1}}\right), \mathrm{K}_{\mathrm{L}}$ é a constante da isoterma de Langmuir $\left(\mathrm{L}_{\mathrm{mg}} \mathrm{m}^{-1}\right)$, $\mathrm{C}_{\mathrm{e}}$ é a concentração do adsorvato no equilíbrio (mg. $\left.\mathrm{L}^{-1}\right)$ e $\mathrm{q}_{\mathrm{e}}$ é a quantidade adsorvida no equilíbrio (mg.g ${ }^{-1}$ ).

Segundo Hall et al. (1966), a característica essencial da isoterma pode ser expressa pela constante adimensional " $\mathrm{R}_{\mathrm{L}}$ ", denominada de fator de separação, a qual permite prever a forma da isoterma de adsorção, indicando se a adsorção é favorável ou desfavorável. O fator de separação pode ser calculado a partir da Equação 4:

$$
R_{L}=\frac{1}{1+K_{L} \cdot C_{0}}
$$

Valore de $R_{L}>1$ indicam que o processo é desfavorável, $\mathrm{R}_{\mathrm{L}}=1$ indica uma isoterma linear, $0<R_{L}<1$ indica que $o$ processo é favorável e $R_{L}=0$ indica que o processo é irreversível (CRINI et al., 2008).

\subsubsection{Isoterma de Freundlich}

O modelo de isoterma de Freundlich é uma equação empírica baseada na adsorção em multicamada, com distribuição não uniforme de calor (EL HADDAD et al., 2014). A expressão da isoterma de Freundlich é representada pela Equação 5 (FREUNDLICH, 1907):

$q_{e}=K_{F} \cdot C_{e}^{1 / n}$

$\mathrm{Na}$ qual $\mathrm{K}_{\mathrm{F}}$ é a constante da isoterma de Freundlich $\left(\mathrm{mg}^{-\mathrm{L}^{-1}}\right)\left(\mathrm{L}_{\mathrm{g}} \mathrm{g}^{-1}\right)^{1 / \mathrm{n}}, \quad \mathrm{C}_{\mathrm{e}} \quad$ é a concentração do adsorvato no equilíbrio $\left(\mathrm{mg} . \mathrm{L}^{-1}\right), 1 / \mathrm{n}$ é o fator de heterogeneidade e 
$\mathrm{q}_{\mathrm{e}}$ é a quantidade adsorvida no equilíbrio (mg.g $\left.{ }^{-1}\right)$.

\subsubsection{Isoterma de Sips}

A isoterma de Sips, também conhecida como isoterma Langmuir-Freundlich, combina os modelos das isotermas de Langmuir e Freundlich, contornando a limitação do aumento da concentração do adsorvato associado ao modelo de Freundlich (FOO et al., 2010). A Equação 6 mostra o modelo da isoterma de Sips:

$q_{e}=\frac{q_{\operatorname{máx}} \cdot K_{S} \cdot C_{e}^{m_{s}}}{1+K_{S} \cdot C_{e}^{m_{s}}}$

Onde $\mathrm{q}_{\text {máx }}$ é a quantidade máxima de adsorção do modelo de Sips (mg.g ${ }^{-1}$ ), $\mathrm{K}_{\mathrm{S}}$ é a constante de equilíbrio do modelo da isoterma de Sips $\left(\mathrm{L} \cdot \mathrm{mg}^{-1}\right), \mathrm{m}_{\mathrm{S}}$ é o expoente do modelo da isoterma de Sips, $\mathrm{C}_{\mathrm{e}}$ é a concentração do adsorvato no equilíbrio (mg. $\left.\mathrm{L}^{-1}\right)$ e $\mathrm{q}_{\mathrm{e}}$ é a quantidade adsorvida no equilíbrio (mg. $\left.\mathrm{g}^{-1}\right)$.

\subsubsection{Isoterma de Redlich-Peterson}

A isoterma de Redlich-Peterson é usada para representar o equilíbrio de adsorção em uma ampla faixa de concentrações e pode ser aplicada em sistemas homogêneos e heterogêneo (PICCIN et al., 2009). O mecanismo de adsorção é um híbrido seguindo uma adsorção em monocamada não ideal (HAMDAOUI et al., 2007). A Equação 7 mostra a isoterma de Redlich-Peterson (REDLICH e PETERSON, 1959):

$$
q_{e}=\frac{K_{R} \cdot C_{e}}{1+a_{R} \cdot C_{e}^{\beta}}
$$

$\mathrm{Na}$ qual $K_{R}$ é a constante do modelo da isoterma de Redlich-Peterson ( $\left(\mathrm{L}^{\mathrm{mg}}{ }^{-1}\right), \mathrm{a}_{\mathrm{R}}$ é a constante da isoterma de Redlich-Peterson $\left(\mathrm{L} . \mathrm{mg}^{-1}\right)^{\beta}, \beta$ é o expoente do modelo da isoterma de Redlich-Peterson, $\mathrm{C}_{\mathrm{e}}$ é a concentração do adsorvato no equilíbrio (mg. $\left.\mathrm{L}^{-1}\right)$ e $\mathrm{q}_{\mathrm{e}}$ é a quantidade adsorvida no equilíbrio (mg.g $\mathrm{g}^{-1}$ ).

\section{RESULTADOS E DISCUSSÃO}

\subsection{Planejamento Experimental}

A Tabela 2 apresenta a matriz de planejamento fatorial $2^{3}$ com a média dos resultados obtidos da variável resposta (qt) em cada combinação de níveis para as variáveis de entrada. Os dados foram tratados no programa Statistica versão 7.0.

Tabela 2 - Matriz de planejamento fatorial $2^{3}$.

\begin{tabular}{|c|c|c|c|c|}
\hline Ensaio & $\mathrm{M}(\mathrm{g})$ & $\mathrm{G}(\mathrm{mm})$ & $\mathrm{A}(\mathrm{rpm})$ & $\mathrm{q}_{\mathrm{t}}(\mathrm{mg} / \mathrm{g})$ \\
\hline 1 & -1 & -1 & -1 & 7,1 \\
\hline 2 & +1 & -1 & -1 & 2,6 \\
\hline 3 & -1 & +1 & -1 & 7,0 \\
\hline 4 & +1 & +1 & -1 & 2,7 \\
\hline 5 & -1 & -1 & +1 & 7,7 \\
\hline 6 & +1 & -1 & +1 & 2,4 \\
\hline 7 & -1 & +1 & +1 & 7,6 \\
\hline 8 & +1 & +1 & +1 & 2,5 \\
\hline
\end{tabular}

Fonte: Autor (2015).

Os resultados apresentados na Tabela 2 foram ajustados a um modelo linear, permitindo a obtenção de correlações empíricas para descrever a capacidade de adsorção do corante azul de metileno a partir do uso da fibra do ouricuri como adsorvente.

O diagrama de pareto, representado pela Figura 3, mostra de forma rápida e clara os efeitos que são estatisticamente significativos. 
Figura 3 - Diagrama de pareto.

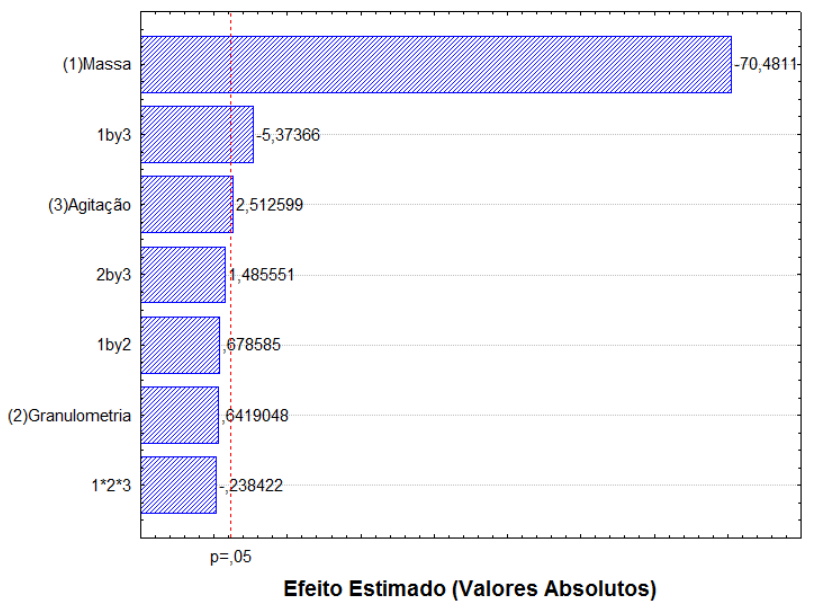

Fonte: Autor (2015).

Analisando a Figura 3, pode-se comprovar que, para a faixa estudada, a variável de maior influência no processo de adsorção foi a massa de adsorvente (M), seguido pelo efeito da interação entre a massa de adsorvente (M) e agitação (A) e a agitação (A).

A partir da análise dos efeitos que foram significativos para a faixa estudada, foi possível a obtenção do modelo empírico. A Equação 8 apresenta o modelo empírico com os parâmetros estatisticamente significativos obtidos pela regressão linear dos dados experimentais.

$q_{t}=4,95-2,4 \cdot x_{M}+0,09 \cdot x_{A}-0,18 \cdot x_{M} x_{A}$

Onde $\mathrm{x}_{\mathrm{M}}$ e $\mathrm{x}_{\mathrm{A}}$ representam as variáveis independentes massa de adsorvente e agitação, respectivamente.

Observa-se pela Equação 8 que o segundo termo, massa de adsorvente (M), representa uma influência negativa sobre a variável resposta $\left(\mathrm{q}_{\mathrm{t}}\right)$ : à medida que se diminui a quantidade de massa de adsorvente obtém-se um aumento na capacidade de adsorção $\left(\mathrm{q}_{\mathrm{t}}\right)$, ou seja, as maiores capacidades de adsorção obtidas para as menores massas de adsorvente podem ser um indicativo de que para o nível superior deste parâmetro (massa de $1,5 \mathrm{~g}$ ) não há saturação do material adsorvente.

Enquanto que no terceiro termo da Equação 8, a velocidade da agitação (A) apresentou um influência positiva, ou seja, quando ocorrer um aumento na velocidade de agitação haverá um aumento na quantidade adsorvida do corante azul de metileno na fibra do ouricuri.

Os resultados obtidos no planejamento experimental foram coerentes, sendo estes similares aos trabalho de Paiva et al. (2014), Rocha et al. (2012) e Oliveira (2010).

Para verificar se o modelo proposto se ajusta aos resultados obtidos, faz-se necessário a construção da tabela da Análise de Variância (ANOVA). A Tabela 3 mostra a avaliação do modelo pela metodologia da ANOVA, a qual indica que o modelo é estatisticamente significativo.

Tabela 3 - Análise de Variância (ANOVA).

\begin{tabular}{|l|c|c|c|}
\hline $\begin{array}{l}\text { Fonte de } \\
\text { Variação }\end{array}$ & $\begin{array}{c}\text { Soma } \\
\text { Quadrática } \\
\text { (SQ) }\end{array}$ & G. L. & $\begin{array}{c}\text { Média } \\
\text { Quadrática } \\
\text { (MQ) }\end{array}$ \\
\hline Regressão & 92,48 & 3 & 30,83 \\
\hline Resíduo & 0,69 & 12 & 0,06 \\
\hline $\begin{array}{l}\text { Falta de } \\
\text { Ajuste }\end{array}$ & 0,54 & 4 & 0,13 \\
\hline Erro Puro & 0,15 & 8 & 0,02 \\
\hline Total & 93,85 & 15 & - \\
\hline $\mathrm{R}^{2}$ & 0,99 & - & - \\
\hline
\end{tabular}

Fonte: Autor (2015).

A Tabela 3 mostra que o coeficiente de correlação $\left(\mathrm{R}^{2}\right)$ foi igual a 0,99 , próximo a unidade, e que os valores de falta de ajuste e erro puro são baixos, o que indica que o modelo foi bem ajustado aos dados experimentais. Além disso, realizando o teste $\mathrm{F}$, para um nível de $95 \%$ de confiança, foi possível verificar que o $\mathrm{F}$ calculado para a regressão das variáveis $\left(\mathrm{F}_{\text {calculado }}=51,4\right)$ é maior que o $\mathrm{F}$ tabelado $\left(\mathrm{F}_{\text {tabelado }}=3,49\right)$, e a 
razão entre o $\mathrm{F}$ calculado e o $\mathrm{F}$ tabelado é maior que $1\left(\mathrm{~F}_{\text {calculado }} / \mathrm{F}_{\text {tabelado }}=14,7\right)$, o que possibilita confirmar a validação do modelo descrito.

A Figura 4 apresenta a superfície de resposta para a quantidade adsorvida, onde pode-se observar que com o aumento da agitação e o decréscimo da massa de adsorvente obtém-se o valor máximo da quantidade de corante azul de metileno adsorvida por massa de fibra do ouricuri.

Figura 4 - Efeito da agitação (A) e massa sobre a quantidade adsorvida.

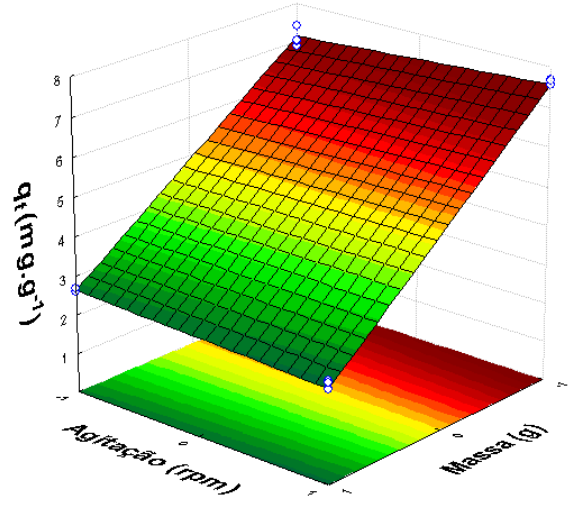

Fonte: Autor (2015).

O valor máximo da quantidade adsorvida foi obtido nas seguintes condições de processo: massa de adsorvente $(\mathrm{M}) 0,5 \mathrm{~g}$, granulometria $(\mathrm{G}) 0,595 \mathrm{~mm}$ e agitação (A) 200 rpm. A partir destas condições operacionais, foram realizados os estudos cinético e de isoterma que estão discutidos nos próximos itens.

\subsection{Cinética de Adsorção}

A Figura 5 apresenta o percentual de remoção do corante azul de metileno em função do tempo de contato utilizando a fibra do ouricuri "in natura".

Pode-se observar que o corante azul de metileno é rapidamente adsorvido pela casca do ouricuri, em torno de 5 minutos, atingindo $90 \%$ da remoção.
Figura 5 - \%Remoção do corante azul de metileno em função do tempo de contato.

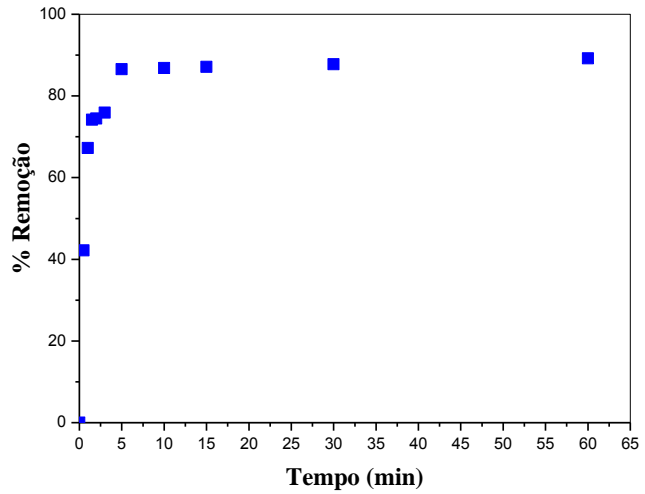

Fonte: Autor (2015).

A cinética rápida caracteriza um processo de adsorção física. Resultados semelhantes foram obtidos por Rocha et al. (2012) e Hameed (2008) que utilizaram a fibra do coco verde como adsorvente, sendo este, material similar a fibra do ouricuri.

\subsection{Isoterma de Adsorção}

A avaliação quantitativa do processo de adsorção foi realizada utilizando isotermas de adsorção, que expressam a relação entre a concentração de equilíbrio em solução $\left(\mathrm{C}_{\mathrm{e}}\right)$ em função da quantidade de corante retido no adsorvente $\left(\mathrm{q}_{\mathrm{e}}\right)$, a uma temperatura constante.

Para determinar o mecanismo e as características da adsorção é necessário encontrar o modelo de isoterma que se ajuste melhor aos dados experimentais. Para este fim, foram utilizados os modelos de isotermas de adsorção na sua forma não-linear, com mostra a Figura 6.

Figura 6 - Isoterma de adsorção do corante azul de metileno na fibra do ouricuri.

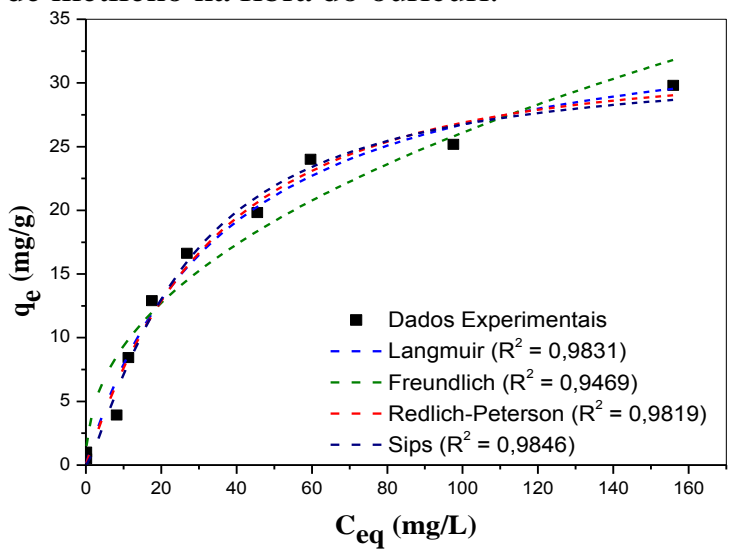

Fonte: Autor (2015). 
A Tabela 4 apresenta a comparação dos resultados obtidos neste trabalho com os da literatura.

Tabela 4 - Resultados encontrados na literatura da capacidade de adsorção máxima, $\mathrm{q}_{\text {máx }}(\mathrm{mg} / \mathrm{g})$.

\begin{tabular}{|l|c|c|}
\hline \multicolumn{1}{|c|}{ Adsorvente } & $\mathrm{q}_{\text {máx }}(\mathrm{mg} / \mathrm{g})$ & \multicolumn{1}{|c|}{ Autor } \\
\hline Pó do coco & 14,36 & Macedo et. al.(2006) \\
\hline $\begin{array}{l}\text { Carvão ativado } \\
\text { do coco }\end{array}$ & 20,62 & Sharma et. al. (2009) \\
\hline $\begin{array}{l}\text { Mesocarpo do } \\
\text { coco verde }\end{array}$ & 21,9 & Rocha et. al. (2012) \\
\hline $\begin{array}{l}\text { Fibra do } \\
\text { ouricuri }\end{array}$ & 29,8 & Este trabalho (2015) \\
\hline
\end{tabular}

Fonte: Autor (2015).

Comparando a fibra do ouricuri, utilizado neste trabalho, com os adsorventes citados na Tabela 4, observa-se que a fibra do ouricuri apresenta um resultado satisfatório na remoção do corante $(29,8 \mathrm{mg} / \mathrm{g})$.

$\mathrm{Na}$ Tabela 5, encontram-se os dados obtidos após aplicação dos modelos de isotermas de Langmuir, Freundlich, RedlichPeterson e Sips para a temperatura de $25^{\circ} \mathrm{C}$.

Tabela 5 - Valores dos parâmetros dos modelos de isoterma de adsorção.

\begin{tabular}{|c|c|c|}
\hline MODELOS & PARÂMETROS & $25^{\circ} \mathrm{C}$ \\
\hline \multirow{4}{*}{ Langmuir } & $\mathrm{q}_{\text {máx }}(\mathrm{mg} / \mathrm{g})$ & 36,4 \\
\hline & $\mathrm{K}_{\mathrm{L}}(\mathrm{L} / \mathrm{mg})$ & 0,03 \\
\hline & $\mathrm{R}_{\mathrm{L}}$ & 0,07 \\
\hline & $\mathbf{R}^{2}$ & 0,983 \\
\hline \multirow{3}{*}{ Freundlich } & $\mathrm{N}$ & 3,3 \\
\hline & $\mathrm{K}_{\mathrm{F}}\left[\left(\mathrm{mg} \cdot \mathrm{L}^{-1}\right)\left(\mathrm{L} \cdot \mathrm{g}^{-1}\right)^{1 / \mathrm{n}}\right]$ & 0,27 \\
\hline & $\mathbf{R}^{2}$ & 0,947 \\
\hline \multirow{4}{*}{ Redlich-Peterson } & $\mathrm{K}_{\mathrm{R}}$ & 0,90 \\
\hline & $a_{R}(L / m g)^{\beta}$ & 0,02 \\
\hline & $\mathrm{B}$ & 1,15 \\
\hline & $\mathbf{R}^{2}$ & 0,982 \\
\hline \multirow{4}{*}{ Sips } & $\mathrm{q}_{\text {máx }}(\mathrm{mg} / \mathrm{g})$ & 31,7 \\
\hline & $\mathrm{K}_{\mathrm{s}}\left(\mathrm{L}^{\prime} \mathrm{mg}\right)$ & 0,02 \\
\hline & $\mathrm{m}_{\mathrm{S}}$ & 1,27 \\
\hline & $\mathbf{R}^{2}$ & 0,985 \\
\hline
\end{tabular}

Fonte: Autor (2015).
A Tabela 5 mostra que o fator de separação $\left(R_{L}=0,07\right)$, calculado com $o$ parâmetro do modelo de Langmuir, indica que o processo é favorável, pois $0<\mathrm{R}_{\mathrm{L}}<1$.

A Figura 6 e a Tabela 5 mostram que os dados experimentais se ajustaram melhor ao modelo de isoterma de Sips, onde o coeficiente de correlação $\left(R^{2}\right)$ foi de 0,985 , e o $\mathrm{q}_{\mathrm{Exp}}=29,8 \mathrm{mg} / \mathrm{g}$ ficou próximo ao $\mathrm{q}_{\text {máx }}=$ $31,7 \mathrm{mg} / \mathrm{g}$.

O modelo de Sips combina elementos das equações de Langmuir e de Freundlich e prevê que, em baixas concentrações de adsorvato, ele se reduz a isoterma de Freundlich, enquanto que, em altas concentrações, se reduz a isoterma de Langmuir, caracterizando adsorção em monocamada.

\section{CONCLUSÃO}

Neste trabalho foi possível investigar a influência das variáveis de processo: massa de adsorvente, granulometria e agitação, através da utilização do planejamento experimental $2^{3}$. Sendo a máxima capacidade de adsorção alcançada com $0,5 \mathrm{~g}$ de fibra do ouricuri, granulometria de $0,595 \mathrm{~mm}$ e velocidade de agitação de $200 \mathrm{rpm}$.

No estudo cinético foi verificado que a adsorção do corante azul de metileno na fibra do ouricuri ocorre rapidamente, em torno de 5 minutos, alcançando $90 \%$ da remoção do corante na solução.

A aplicação dos modelos das isotermas de adsorção mostrou que o processo é favorável, segundo o fator de separação $\left(R_{L}\right)$, e os dados experimentais se ajustaram melhor ao modelo de Sips, o que pode indicar uma adsorção em monocamada em altas concentrações.

A fibra do ouricuri se apresentou um ótimo adsorvente alternativo por ser um subproduto agroindustrial sem valor agregado e com boa capacidade de adsorção. 


\section{NOMENCLATURA}

$1 / \mathrm{n} \quad$ fator de heterogeneidade

$\beta$ expoente do modelo da isoterma de Redlich-Peterson

$\mathrm{a}_{\mathrm{R}}$ constante da isoterma de RedlichPeterson $\left(\mathrm{L} \cdot \mathrm{mg}^{-1}\right)^{\beta}$

$\mathrm{C}_{0}$ concentração inicial do adsorvato (mg.L $\mathrm{L}^{-1}$ )

$\mathrm{C}_{\mathrm{e}}$ concentração do adsorvato no equilíbrio (mg. $\left.\mathrm{L}^{-1}\right)$

$\mathrm{C}_{\mathrm{f}} \quad$ concentração final da solução $\left(\mathrm{mg} . \mathrm{L}^{-1}\right)$

G.L. graus de liberdade

$\mathrm{K}_{\mathrm{F}}$ constante da isoterma de Freundlich $\left(\mathrm{mg} . \mathrm{L}^{-1}\right)\left(\mathrm{L} \cdot \mathrm{g}^{-1}\right)^{1 / \mathrm{n}}$

$\mathrm{K}_{\mathrm{L}}$ constante da isoterma de Langmuir (L.mg ${ }^{-1}$ )

$\mathrm{K}_{\mathrm{R}}$ constante do modelo da isoterma de Redlich-Peterson (L.mg ${ }^{-1}$ )

$\mathrm{K}_{\mathrm{S}}$ constante de equilíbrio do modelo da isoterma de Sips (L.mg ${ }^{-1}$ )

M massa de adsorvente $(\mathrm{g})$

$\mathrm{m}_{\mathrm{S}}$ expoente do modelo da isoterma de Sips

$\mathrm{q}_{\mathrm{e}} \quad$ quantidade adsorvida no equilíbrio (mg/g)

$\mathrm{q}_{\text {máx }}$ quantidade máxima de cobertura em monocamada $\left(\mathrm{mg} \cdot \mathrm{g}^{-1}\right)$

$\mathrm{R}_{\mathrm{L}} \quad$ Fator de separação

$\mathrm{V}$ volume da solução (L)

\section{REFERÊNCIAS}

AHMAD, A. et al. Removal of $\mathrm{Cu}($ II) and $\mathrm{Pb}$ (II) ions from aqueous solutions by adsorption on sawdust of Meranti wood. Desalination, v. 247, p.636-646, 2009.

BORBA, C.E. et al. Estudo da cinética e do equilíbrio de adsorção dos corantes azul turquesa QG e amarelo reativo $3 R$ em carvão ativado. Engevista, v. 14, p. 135-142, 2012.

BRITO, S. M. O. et al. Brazil nut shells as a new biosorbent to remove methylene blue and indigocarmine from aqueous solutions. Journal of Hazardous Materials, v.174, p. 8492, 2010.

CONAMA - Conselho Nacional do Meio Ambiente - Ministério do Desenvolvimento Urbano e Meio Ambiente. Resolução n ${ }^{\circ}$ 357, de 25 de abril de 2005.

CRINI, G.; BADOT, P. M. Aplication of chitosan, a natural aminopolysaccharide, for dye removal from aqueous solutions by adsorption processes using batch studies: A review of recent literature. Progress in Polymer Science, v. 33, p. 399-447, 2008.

DONATO, A. Licuri-Ouro Verde do semíÁrido. Jornal A Tarde Salvador.

EL HADDAD, M. et al. Calcined mussel shells as a new and eco-friendly to remove textile dyes from aqueous solutions. Journal of the Taiwan Institute of Chemical Engineers, v. 45, p. 533-540, 2014.

EMBRABA. Palmeira típica do sertão nordestino. Disponível em: <http://www. agrocim.com.br/>. Acesso em: 10 jun. 2015.

FOO, K. Y.; HAMEED, B. H. Insights into the modeling of adsorption isotherm systems. Chemical Engineering Journal, v.156, p. 2-10, 2010.

FREUNDLICH, H. Uber die adsorption in Losungen. Z. Phys. Chem., v. 57, p. 385, 1907.

GAO, J. et al. Binary biosorption of acid red 14 and reactive red 15 onto acid treated okara: Simultaneous spectrophotometric determination of two dyes using partial least squares regression. Chemical Engineering Journal, v. 171, p. 967975, 2011. 
GIMBERT, F. et al. Adsorption isotherm models for dye removal by cationized starch-based material in a single component system: Error analysis. Journal of Hazardous Materials, v. 157, p. 34-46, 2008.

GULER, U.A.; SARIOGLU, M. Single and binary biosorption of $\mathrm{Cu}(\mathrm{II}), \mathrm{Ni}(\mathrm{II})$ and methylene blue by raw and pretreated Spirogyra sp.: Equilibrium and kinetic modeling. J. Environ. Chem. Eng., v. 1, p. 369-377, 2013.

HALL, K. R. et al. Pore and solid-diffusion kinetics in fixed-bed adsorption under constant-pattern conditions. Industrial and Engineering Chemistry, Fundamentals, v. 5, p. 212-219, 1966.

HAMDAOUI, O.; NAFFRECHOUX, E. Modeling of adsorption isotherms of phenol and chlorophenols onto granular activated carbon Part II. Models with more than two parameters. Journal of Hazardous Materials, v. 147, p. 401-411, 2007.

HAMEED, B. H.; MAHMOUND, D. K.; AHMAD, A. L. Equilibrium modeling and kinetic studies on the adsorption of basic dye by a low-cost adsorbent: Coconut (Cocos nucifera) bunch waste. Journal of Hazardous Materials, v. 158, p. 65-72, 2008.

LANGMUIR, I. The adsorption of gases on plane surfaces of glass, mica and platinum. Journal of the American Chemical Society, v.40, p. 1361-1403, 1918.

OLIVEIRA, E. H. C. Adsorção de corantes da indústria têxtil (Indosol) em resíduos industriais (Lama vermelha e Argila Esmectita). 2010. 73p. Dissertação (Mestrado em Engenharia Química) - Universidade Federal de Pernambuco, Recife, 2010.
PAIVA, T. M. N. Estudo comparativo de adsorção de corante têxtil utilizando carvão ativado e conchas de ostras crassostrea rhizophorae. XX Congresso Brasileiro de Engenharia Química - COBEQ 2014, Florianópolis, SC, 2014.

PAZ, D. S. et al. Use of papaya seeds as a biosorbent of methylene blue from aqueous solution. Water Science and Technology, v. 68, p. 441-447, 2013.

PICCIN, J. S. et al. Adsorption of FD\&C Red No. 40 by chitosan: Isotherms analysis. Journal of Food Engineering, v. 95, p. 16-20, 2009.

REDLICH, O.; PETERSON, D. L. A useful adsorption isotherm. Journal Phys. Chem., v. 63, p. 1024, 1959.

ROCHA, O. R. S. Avaliação do processo adsortivo utilizando o mesocarpo de coco verde para remoção do corante cinza reativo BF-2R. Química Nova, v. 35, n. 7, p. 1369-1374, 2012.

TAN, I.A.W.; AHAMAD, A.L.; HAMEED, B.H. Adsorption of basic dye usin activated carbon prepared from oil palm shell: batch and fixed bed studies. Desalination, v. 225, p. 13-28, 2008.

TANYILDIZI, M. Ş. Modeling of adsorption isotherms and kinetics of reactive dye from aqueous solution by peanut hull. Chemical Engineering Journal, v. 168, p.1234-1240, 2011.

YAGUB, M. T. et al. Dye and its removal from aqueous solution by adsorption: A review. Advances in Colloid and Interface Science, v. 209, p. 172-184, 2014.

AGRADECIMENTOS CAPES e UFAL. 